\title{
Heat Stress Prevents the Decrease in Succinate Dehydrogenase Activity in the Extensor Digitorum Longus of Streptozotocin-Induced Diabetic Rats
}

\author{
K. NONAKA ${ }^{1}$, S. UNE ${ }^{2}$, M. KOMATSU ${ }^{3}$, R. YAMAJI ${ }^{4}$, J. AKIYAMA ${ }^{3}$ \\ ${ }^{1}$ Department of Physical Therapy, School of Comprehensive Rehabilitation, Osaka Prefecture \\ University, Habikino, Japan, ${ }^{2}$ Department of Living Science, Shujitsu Junior College, Okayama, \\ Japan, ${ }^{3}$ Department of Physical Therapy, School of Health Care and Social Welfare, Kibi \\ International University, Takahashi, Japan, ${ }^{4}$ Division of Applied Life Sciences, Graduate School of \\ Life and Environmental Sciences, Osaka Prefecture University, Sakai, Japan
}

Received February 14, 2017

Accepted August 24, 2017

On-line November 10, 2017

\begin{abstract}
Summary
This study aimed to investigate whether heat stress (HS) prevents a decrease in succinate dehydrogenase (SDH) activity and heat shock protein 60 (HSP60) and superoxide dismutase 2 (SOD2) contents in the extensor digitorum longus of streptozotocin (STZ)-induced diabetic rats. Twelve-week-old male Wistar rats were assigned to one of the four groups ( $n=6 /$ group): control (Con), HS, diabetes mellitus (DM), and diabetes mellitus and heat stress $(\mathrm{DM}+\mathrm{HS})$. Diabetes was induced by the administration of STZ $(50 \mathrm{mg} / \mathrm{kg})$. HS was initiated 7 days after STZ treatment and performed at $42^{\circ} \mathrm{C}$ for 30 min 5 times a week for 3 weeks. SDH activity was decreased in the DM and $\mathrm{DM}+\mathrm{HS}$ groups. However, SDH activity was greater in the $\mathrm{DM}+\mathrm{HS}$ group than in the DM group. Although HSP60 content was lower in the DM group than in the Con group, it was maintained in the DM+HS groups and was higher than that in the DM group. SOD2 content was decreased only in the DM group. These findings suggest that HS prevents the decrease in $\mathrm{SDH}$ activity in the skeletal muscle induced by DM. According to this mechanism, the maintenance of SOD2 and HSP60 by HS may suppress the increase in oxidative stress.
\end{abstract}

\section{Key words}

Diabetes mellitus $\bullet$ Heat stress $\bullet$ Succinate dehydrogenase $\bullet$ Heat shock protein 60 - Superoxide dismutase 2

\section{Corresponding author}

K. Nonaka, Faculty of Health Sciences, Kyoto Tachibana
University, 34 Oyakeyamada-cho, Yamashina-ku, Kyoto, 6078175, Japan. E-mail: nonaka-k@tachibana-u.ac.jp

\section{Introduction}

Diabetes mellitus (DM) is accompanied by a decrease in succinate dehydrogenase (SDH) activity, which is an important mitochondrial enzyme involved in energy metabolism, in skeletal muscle (He et al. 2001). Abnormalities of skeletal muscle energy metabolism, especially mitochondrial dysfunction, are associated with lowered exercise capacity (Okita et al. 1998, Yokota et al. 2011), which was also observed in patients with DM (Gürdal et al. 2015). Lowered exercise capacity in patients with DM is a predictor of mortality (Church et al. 2004). Hence, it is important for patients with DM to maintain SDH activity in skeletal muscle to prevent declines in exercise capacity.

Exercise, which is a standard intervention for DM patients, has a positive effect on SDH in skeletal muscle, but we believe that exercise can be problematic for some patients. Little et al. (2011) have reported that exercise increased respiratory complex II (known as $\mathrm{SDH}$ ) in skeletal muscle of DM patients. However, the ability to perform moderate exercise could be limited by lowered exercise capacity induced by DM. Accordingly, the development of an alternative therapy for maintaining skeletal muscle mitochondrial function in patients with 
DM is essential. Heat stress (HS) and heat stress proteins (HSPs), which are molecular chaperones activated in response to HS, have recently attracted for their positive effects on skeletal muscle. HS has been reported to increase HSPs and maintain mitochondrial function in denervated skeletal muscle from mice (Tamura et al. 2015). We previously reported that HS attenuated muscle atrophy in the extensor digitorum longus (EDL) of streptozotocin (STZ)-induced diabetic rats (Nonaka et al. 2015). Mitochondrial toxicity could be the key mediator of skeletal muscle atrophy (Powers et al. 2012, Tryon et al. 2014). These findings suggest that HS can potentially prevent the decrease in SDH activity in skeletal muscle induced by DM. Therefore, HS could be used as an alternative to exercise for preventing the decrease in skeletal muscle. However, it is unknown whether HS has a preventive effect on the decrease in SDH activity in skeletal muscle induced by DM.

We hypothesized that HS prevents decreases in SDH activity in the skeletal muscle of diabetic rats. Therefore, the purpose of this study was to investigate the effect of HS on SDH activity in the EDL of diabetic rats. The EDL was selected in this study because it is predominantly composed of type II fibers (Soukup et al. 2002), and the loss of SDH activity induced by DM has been reported to predominantly occur in type II fibers than in type I fibers (Armstrong et al. 1975). Moreover, levels of superoxide dismutase 2 (SOD2; known as MnSOD), which is an antioxidant enzyme located in mitochondria, were measured, as it has been reported that SOD2 is associated with SDH activity in skeletal muscle (Lustgarten et al. 2011) and that SOD2 content is decreased in the skeletal muscle of diabetic rats (Malardé et al. 2015, Guan et al. 2016).

\section{Methods}

Animals

Twenty-four male Wistar rats (12 weeks old) were housed in a temperature-controlled room maintained at $22{ }^{\circ} \mathrm{C}$ under a 12 -h light-dark cycle and allowed free access to food and water. The rats were randomly divided into four groups consisting of six animals per group as follows: control (Con), HS, diabetes mellitus (DM), and diabetes mellitus and heat stress (DM+HS). Diabetes was induced by a single intraperitoneal injection of STZ ( $50 \mathrm{mg} / \mathrm{kg}$ body weight; Wako Pure Chemical Industries, Ltd., Osaka, Japan) dissolved in physiological saline according to a previous study (Nonaka et al. 2014).
Equivalent amounts of the vehicle were injected into rats in the non-diabetic groups. Blood was collected from the tail vein, and non-fasting blood glucose concentrations were measured using the Glutest Ace R (Sanwa Kagaku Kenkyusho, Co., Ltd., Nagoya, Japan) 7 days after the STZ injection. Rats with blood glucose concentrations $>300 \mathrm{mg} / \mathrm{dl}$ were considered to have diabetes.

Twenty-four hours after the final HS, rats were euthanized via an intraperitoneal injection of an overdose of sodium pentobarbital $(150 \mathrm{mg} / \mathrm{kg})$. The EDL was removed, weighed immediately, frozen in isopentane cooled by liquid nitrogen and stored at $-80^{\circ} \mathrm{C}$ until further analysis. The muscle weight/body weight ratio was also calculated because muscle weight is affected by body weight. The study was approved by the Institutional Animal Care and Use Committee of Osaka Prefecture University.

\section{HS protocol}

HS was performed according to a previously reported method (Nonaka et al. 2015). The lower half of body of each rat was immersed in hot water at $42{ }^{\circ} \mathrm{C}$ for 30 min under anesthesia induced by an intraperitoneal injection of sodium pentobarbital $(50 \mathrm{mg} / \mathrm{kg})$. HS was started 7 days after the injection of STZ and was performed once daily five times a week for 3 weeks. To avoid any confounding effects of the anesthesia, the rats in the Con and DM groups were anesthetized in a similar manner as those in the HS and DM+HS groups.

\section{Western blot}

The muscles were homogenized in ice-cold extraction buffer (20 mM Tris-HCl, pH 7.4, $25 \mathrm{mM} \mathrm{KCl}$, $1 \%$ Triton $\mathrm{X}-100)$ containing cOmplete $^{\mathrm{TM}}$ protease inhibitor cocktail (Roche Diagnostics, Tokyo, Japan). Homogenates were centrifuged at $12,000 \times \mathrm{g}$ for $10 \mathrm{~min}$ at $4{ }^{\circ} \mathrm{C}$, and aliquots of supernatants were used for subsequent analyses. The protein concentrations of the aliquots were measured using a Coomassie Protein Assay Kit (Thermo Fisher Scientific K.K., Yokohama, Japan). The absorbance was read at $595 \mathrm{~nm}$ using a microplate reader (Model 680; Bio-Rad Laboratories, Inc., CA, USA) to determine the protein concentration. The aliquots were diluted with extraction buffer, and EzApply (ATTO, Tokyo, Japan) was added to the diluted aliquots. The final protein concentration of the samples for Western blot analysis was adjusted to $2 \mu \mathrm{g} / \mu \mathrm{l}$, and samples were boiled for $5 \mathrm{~min}$. The samples were applied to a 5-20\% gradient polyacrylamide gel (ATTO, Tokyo, Japan), and 
electrophoresis was performed at a constant current of $20 \mathrm{~mA} / \mathrm{gel}$ for $60 \mathrm{~min}$. The separated proteins were then transferred to PVDF membranes (ATTO, Tokyo, Japan) at a constant current of $2 \mathrm{~mA} / \mathrm{cm}^{2}$ for $60 \mathrm{~min}$ via the semi-dry blotting method. The membranes were stained with Ponceau-S staining solution (Beacle, Inc., Kyoto, Japan) and scanned. They were destained and blocked with EzBlock Chemi (ATTO) for $1 \mathrm{~h}$ at room temperature and subsequently incubated overnight at $4{ }^{\circ} \mathrm{C}$ with the following primary antibodies: anti-HSP60 (1:5,000; SMC-110; Stress Marq Biosciences Inc., Victoria, Canada) and anti-SOD2 (1:5,000; sc-30080; Santa Cruz Biotechnology, Inc., CA, USA). Following incubation, the membranes were washed three times for $10 \mathrm{~min} /$ wash in EzWash (ATTO) containing $0.1 \%$ Tween 20 (TTBS) and reacted for $1 \mathrm{~h}$ at room temperature with the following secondary horse radish peroxidase-conjugated antibodies: anti-mouse IgG (1:25,000; 01803-44; Nacalai Tesque, Kyoto, Japan) for HSP60 and anti-rabbit IgG $(1: 25,000$; 01827-44; Nacalai Tesque) for SOD2. Following this incubation, the membranes were washed three times for $10 \mathrm{~min} /$ wash in TTBS and reacted with ECL ${ }^{\mathrm{TM}}$ Prime Western Blot Detection Reagent (CE Healthcare UK Ltd., Buckinghamshire, England) for $5 \mathrm{~min}$ at room temperature, and the protein bands were obtained using LumiCube (Liponics, Inc., Tokyo, Japan). The protein bands were analyzed using JustTLC software (Sweday, Sondra Sandby, Sweden). The 45-kDa bands from the Ponceaustained membranes were used as protein loading controls (Perry et al. 2016). The data were normalized to the Con value.

\section{Histochemistry}

Muscles were sliced into $10-\mu \mathrm{m}$-thick cross-sections using a cryostat at $-20{ }^{\circ} \mathrm{C}$. The tissue sections were dried at room temperature for $30 \mathrm{~min}$.

SDH staining was performed as described previously with minor modifications (Ishihara et al. 2015). The tissue sections were incubated in $0.1 \mathrm{M}$ phosphate buffer ( $\mathrm{pH}$ 7.6) containing $0.9 \mathrm{mM}$ sodium azide, $0.9 \mathrm{mM}$ 1-methoxyphenazine methylsulphate, $1.5 \mathrm{mM}$ nitroblue tetrazolium, $5.6 \mathrm{mM}$ EDTA and $48 \mathrm{mM}$ succinate disodium salt for $45 \mathrm{~min}$ at $37^{\circ} \mathrm{C}$. The stained sections were observed under a microscope at $\times 100$ magnification, and a randomly selected image was obtained using a digital camera. The SDH activity of muscle fibers was measured as the mean optical density using ImageJ software (NIH, Bethesda, USA), and the mean SDH activity of muscle fibers was determined as the measured values.
For immunohistochemical staining of slow-twitch muscle fibers, the tissue sections were fixed in $10 \%$ formalin solution for $15 \mathrm{~min}$ at $4{ }^{\circ} \mathrm{C}$, and immunohistochemical staining was performed using a VECTASTAIN $^{\circledR} \quad$ Universal quick kit (Vector Laboratories, CA, USA) according to the manufacturer's instructions. Endogenous peroxidase was quenched in $0.3 \% \mathrm{H}_{2} \mathrm{O}_{2}$ in methanol for $30 \mathrm{~min}$. The sections were incubated with blocking serum for $10 \mathrm{~min}$ and reacted with mouse monoclonal anti-slow myosin (1:400; GTX11083; Gene Tex, Inc., Irvine, CA, USA) for $2 \mathrm{~h}$ at room temperature. The slides were washed three times in PBS for $5 \mathrm{~min} /$ wash, and the sections were incubated in biotinylated universal secondary antibody working solution for $10 \mathrm{~min}$. The sections were washed three times in PBS for $5 \mathrm{~min} / \mathrm{wash}$ and incubated in streptavidin/peroxidase complex working solution for 5 min. The sections were washed three times in PBS for $5 \mathrm{~min} / \mathrm{wash}$, and slow-twitch muscle fibers were visualized using diaminobenzidine substrate. The stained sections were observed under a microscope at $\times 100$ magnification, and two randomly selected images were obtained using a digital camera. The fast- and slow-twitch muscle fiber cross-sectional areas (CSAs) were measured using ImageJ software, and the means were determined. The muscle fiber CSA/body weight ratio was also calculated because muscle fiber CSAs are believed to be affected by body weight.

\section{Statistical analysis}

All data were expressed as the mean $\pm \mathrm{SEM}$. Statistical analysis was performed via comparisons among the four experimental groups. The Shapiro-Wilk test was used to verify whether the data were normally distributed. For non-normally distributed data, namely the data for blood glucose levels and muscle weight, the Kruskal-Wallis test was used. The Steel-Dwass post hoc test was performed to determine the significance of differences among the four groups. For normally distributed data, Levene's test of homogeneity of variance was performed. For data that did not exhibit homogeneous variance, namely that for the initial body weight, Welch's one-way ANOVA was used. For data exhibiting homogeneous variance, one-way ANOVA was used. Tukey's post hoc test was performed to determine the significance of differences among the four groups. $p<0.05$ was considered significant. Statistical analyses were performed using $\mathrm{R}$ software ver. 2.8.1 and $\mathrm{R}$ commander software package ver.1.4-8 (The R project 
for statistical computing, https://www.r-project.org/).

\section{Results}

\section{Body weight and blood glucose}

Body weight and blood glucose data are shown in Table 1. The initial body weights of rats were similar among the four groups, whereas the final body weights of rats were decreased in the DM and DM+HS groups compared with that in the Con group $(p<0.001)$.
Furthermore, the final body weights of rats were lower in the HS and DM+HS groups than in the Con and DM groups, respectively $(p<0.001$ and $p<0.01$, respectively). These findings suggest that HS induces decreases in rat body weight.

Blood glucose content was significantly higher in the DM and DM+HS groups than in the Con and HS groups 7 days after the STZ injection $(p<0.05)$. The blood glucose level exceeded $300 \mathrm{mg} / \mathrm{dl}$ in all rats in the $\mathrm{DM}$ and $\mathrm{DM}+\mathrm{HS}$ groups.

Table 1. Body weights and blood glucose levels at 7 days after STZ injection in the four groups.

\begin{tabular}{lcccc}
\hline & Con & HS & DM & DM+HS \\
\hline Initial body weight $(g)$ & $286.3 \pm 2.2$ & $289.7 \pm 4.4$ & $294.7 \pm 7.1$ & $288.7 \pm 2.2$ \\
Final body weight $(g)$ & $334.0 \pm 3.5$ & $298.0 \pm 6.3^{* * *}$ & $248.5 \pm 5.0^{* * * \# \#}$ & $217.7 \pm 4.6^{* * * \# \#+\dagger}$ \\
Blood glucose $(\mathrm{mg} / \mathrm{dl})$ & $145.8 \pm 26.1$ & $114.5 \pm 10.5$ & $449.8 \pm 20.9^{* \#}$ & $511.7 \pm 29.7$ *\# $^{*}$ \\
\hline
\end{tabular}

Values are presented as means \pm SEM. $n=6$ per group. STZ, streptozotocin, Con, control; DM, diabetes; DM+HS, diabetes and heat stress; HS, heat stress. ${ }^{*} p<0.05$ and ${ }^{* * *} p<0.001$ vs. the Con group. ${ }^{\#} p<0.05$ and ${ }^{\# \# \#} p<0.001$ vs. the HS group. ${ }^{++} p<0.01$ vs. the DM group.

\section{SDH activity}

SDH activity was measured by histochemical staining in the skeletal muscle of diabetic rats. SDH-stained images and activity are shown in Figure 1. Compared with the Con group data, SDH activity was significantly decreased in the DM $(48 \%$ of Con, $p<0.001)$ and $\mathrm{DM}+\mathrm{HS}$ groups ( $72 \%$ of Con, $p<0.01$ ). However, SDH activity in the EDL was significantly lower in the DM group than in the DM+HS group (68\% of DM+HS, $p=0.032$ ). These findings suggest that HS prevents mitochondrial dysfunction in the skeletal muscle of diabetic rats.

\section{HSP60 content}

HSP60, which was induced by HS, were measured by Western blot, as shown in Figure 2. Compared with the Con group levels, HSP60 levels in the EDL were significantly increased in the HS group (134\% of Con, $p<0.001)$. These results suggest that HS sufficiently induced physiological and biochemical responses, as HSPs are biomarkers induced by HS (Oishi et al. 2002). Compared with the Con group level, HSP60 content in the EDL was significantly decreased in the DM group $(49 \%$ of Con, $p<0.001)$ but not in the $\mathrm{DM}+\mathrm{HS}$ group $(p=0.18)$. Moreover, HSP60 content in the EDL was significantly higher in the DM+HS group than in the DM group $(176 \%$ of DM, $p<0.001)$. These findings suggest that HS prevents decreases in the HSP60 content in the EDL of diabetic rats.

\section{SOD2 content}

SOD2 content was measured by Western blotting, as SOD2 is reportedly associated with SDH activity in skeletal muscle (Lustgarten et al. 2011). SOD2 content is shown in Figure 3. Compared with the Con group level, SOD2 content was significantly decreased in the DM group (59\% of Con, $p=0.042$ ) but not in the DM+HS group $(p=0.36)$. These findings suggest that HS prevents decreases in SOD2 content in the skeletal muscle of diabetic rats.

Muscle weight, muscle weight/body weight, and muscle fiber CSA

Muscle weight, muscle weight/body weight ratio, muscle fiber CSAs, and muscle fiber CSA/body weight were measured to verify the degree of skeletal muscle atrophy in diabetic rats. These data are shown in Table 2. Muscle weight was significantly decreased in the DM ( $64 \%$ of Con, $p=0.02)$ and DM+HS groups ( $62 \%$ of Con, $p=0.02$ ) compared with that in the Con group. Compared with the Con group ratio, the muscle weight/body weight ratio was significantly decreased in the DM group ( $91 \%$ of Con, $p=0.046$ ) but not in the DM+HS group $(p=0.54)$. 


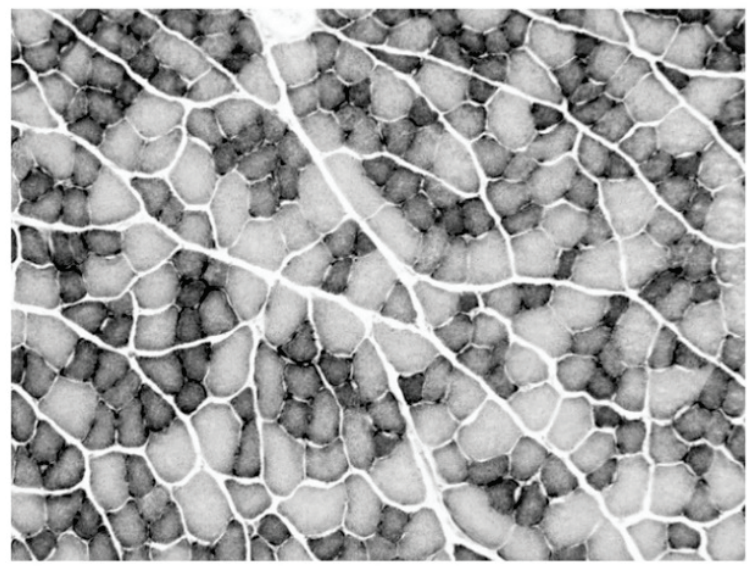

Con

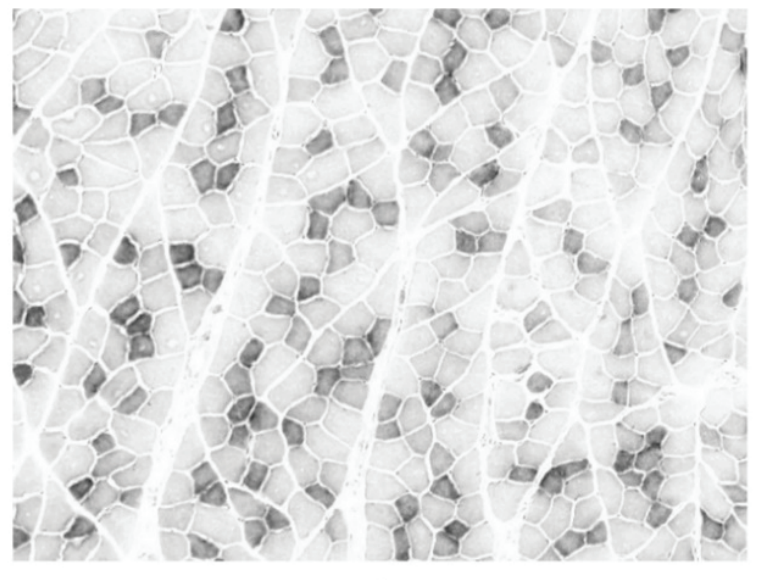

DM

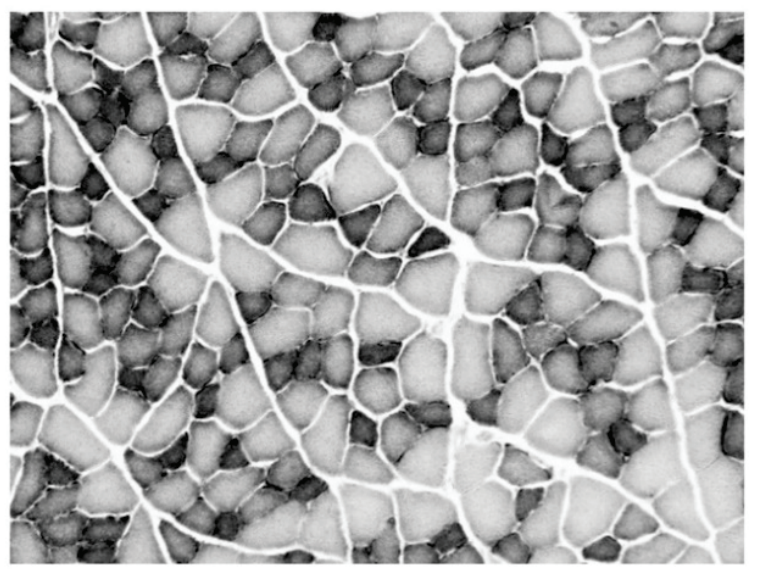

HS

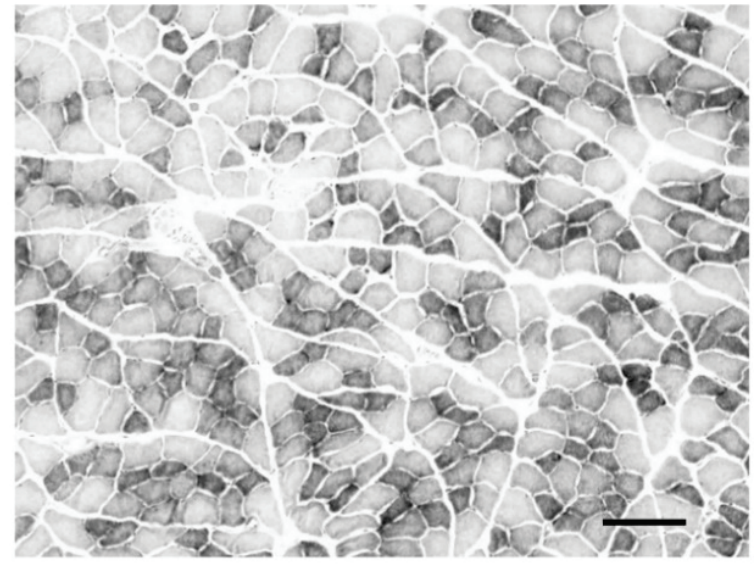

$\mathrm{DM}+\mathrm{HS}$

(a)

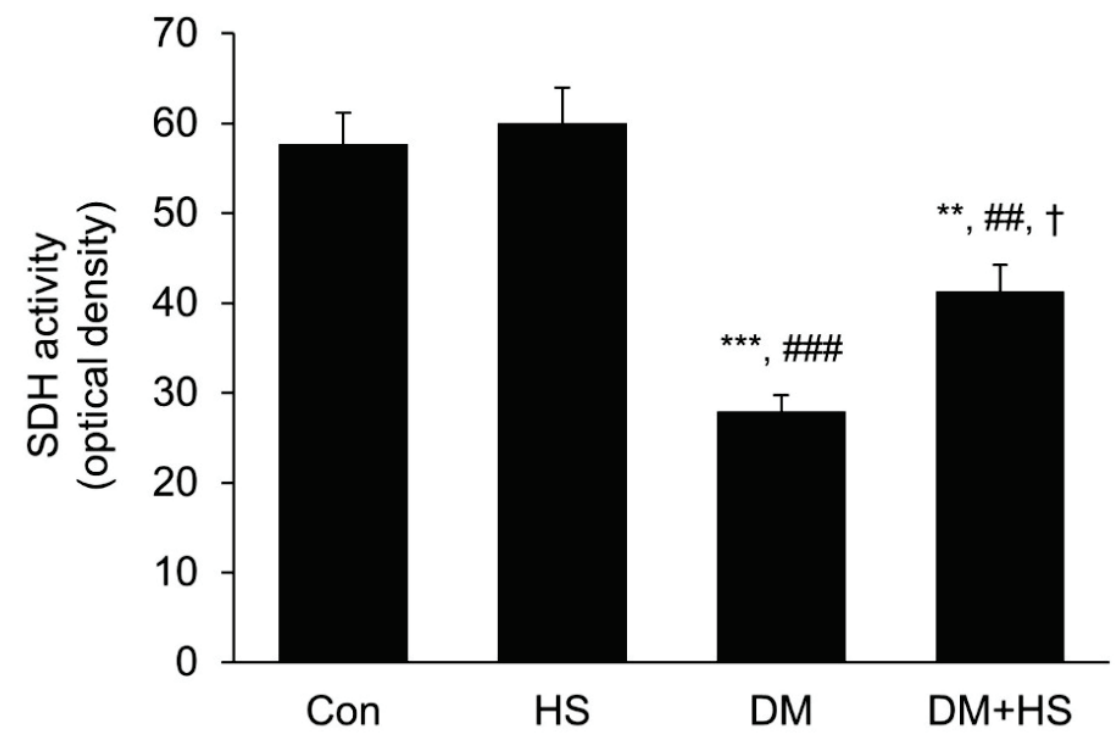

(b)

Fig. 1. Succinate dehydrogenase (SDH)-stained cross-sections of the extensor digitorum longus (EDL) (a). Graphical representation showing the mean SDH activity of the EDL in the four groups (b). Values are expressed as means $\pm \mathrm{SEM}$. $\mathrm{n}=6$ per group. ${ }^{* *} p<0.01$ and $* * * p<0.001$ vs. the Con group; ${ }^{\# \#} p<0.01$ and ${ }^{\# \# \#} p<0.001$ vs. the HS group; ${ }^{\dagger} p<0.05$ vs. the DM group. Scale bar $=100 \mu \mathrm{m}$. Con, control; DM, diabetes; DM+HS, diabetes and heat stress; HS, heat stress. 


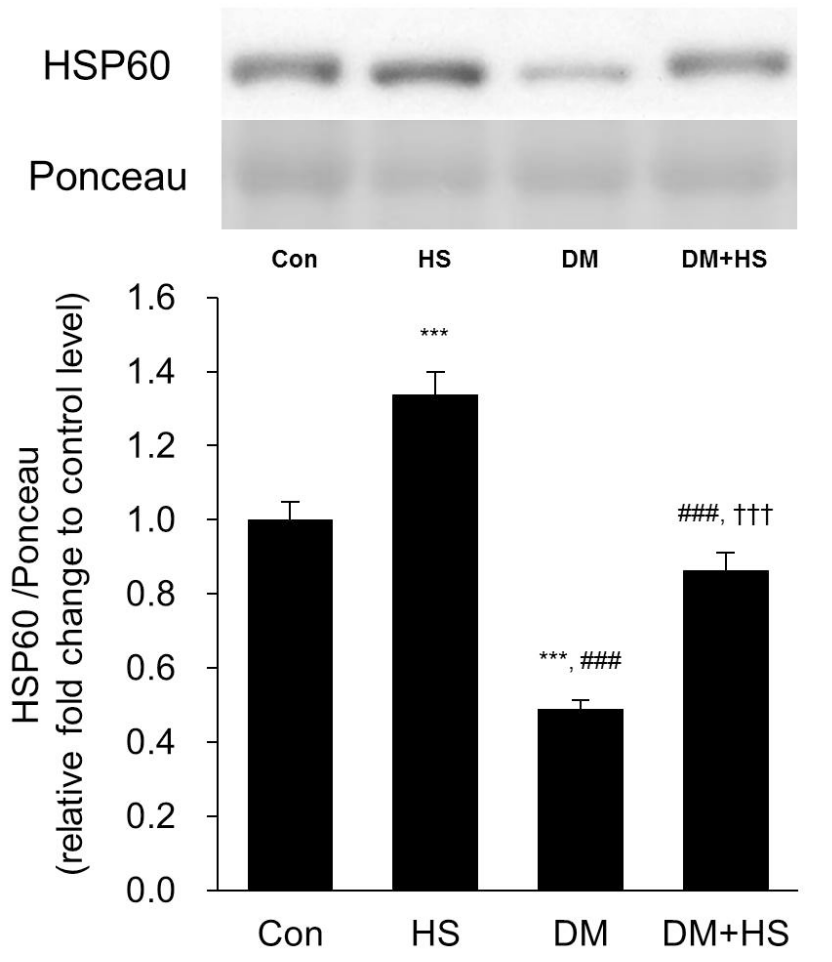

Fig. 2. Expression levels of heat shock protein 60 (HSP60) in the extensor digitorum longus (EDL). The expression pattern and protein concentrations of HSP60 in the EDL in the four groups are presented. Values are expressed as means \pm SEM. $n=6$ per group. ${ }^{* * *} p<0.001$ vs. the Con group; ${ }^{\# \#} p<0.001$ vs. the HS group; ${ }^{+++} p<0.001$ vs. the DM group. Con, control; DM, diabetes; DM+HS, diabetes and heat stress; HS, heat stress.

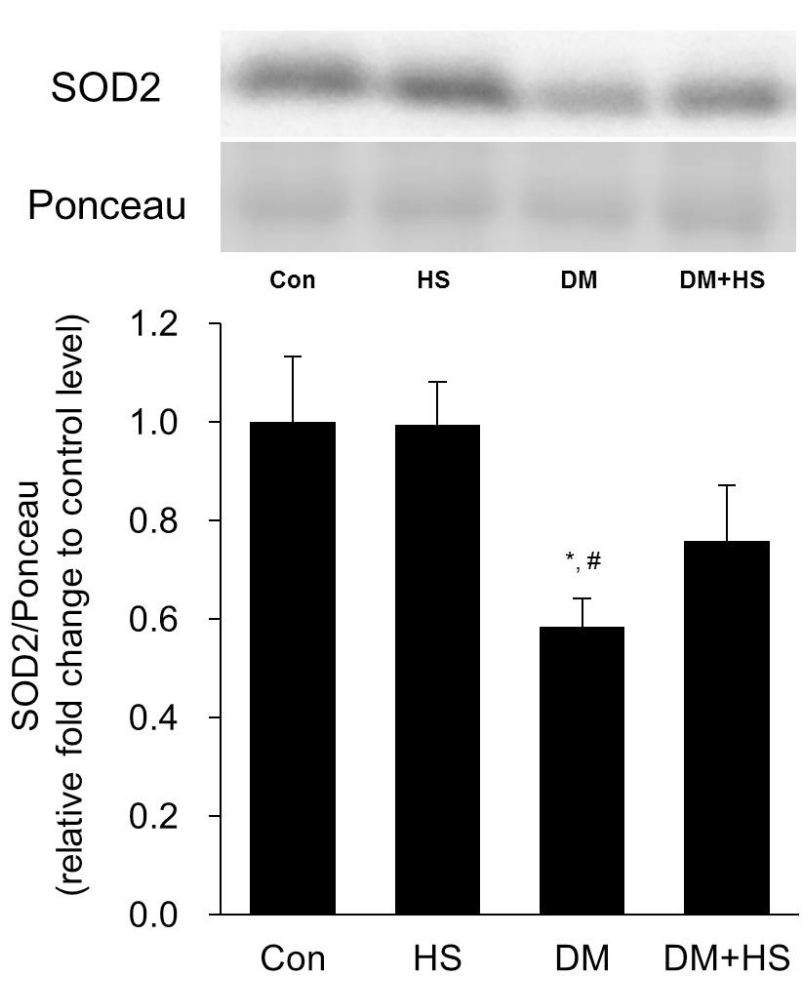

Fig. 3. Expression levels of superoxide dismutase 2 (SOD2) in the extensor digitorum longus (EDL). The expression pattern and protein concentrations of SOD2 in the EDL in the groups are shown. Values are expressed as means \pm SEM. $n=6$ per group. ${ }^{*} p<0.05$ vs. the Con group; ${ }^{\#} p<0.05$ vs. the HS group. Con, control; DM, diabetes; DM+HS, diabetes and heat stress; HS, heat stress.

Table 2. EDL muscle weights and muscle fiber CSAs in the four groups.

\begin{tabular}{lcccc}
\hline & Con & HS & DM & DM+HS \\
\hline Muscle weight $(\mathrm{mg})$ & $137.3 \pm 1.8$ & $129.7 \pm 2.1$ & $87.5 \pm 1.7^{* \#}$ & $85.3 \pm 2.5^{* \#}$ \\
Muscle weight/body weight $(\mathrm{mg} / \mathrm{g})$ & $0.411 \pm 0.008$ & $0.436 \pm 0.008$ & $0.372 \pm 0.007^{* \# \#}$ & $0.393 \pm 0.013^{\#}$ \\
Fast-twitch muscle fiber CSA $\left(\mu \mathrm{m}^{2}\right)$ & $1419 \pm 108$ & $1516 \pm 67$ & $672 \pm 28^{* * * \# \#}$ & $821 \pm 82^{* * * \# \#}$ \\
Fast-twitch muscle fiber CSA/body weight $\left(\mu \mathrm{m}^{2} / \mathrm{g}\right)$ & $4.24 \pm 0.30$ & $5.09 \pm 0.22$ & $2.71 \pm 0.10^{* * \# \#}$ & $3.76 \pm 0.34^{\#+}$ \\
Slow-twitch muscle fiber CSA $\left(\mu \mathrm{m}^{2}\right)$ & $610 \pm 79$ & $668 \pm 36$ & $377 \pm 18^{*}$ \#\#\# & $459 \pm 45^{\#}$ \\
Slow-twitch muscle fiber CSA/body weight $\left(\mu \mathrm{m}^{2} / \mathrm{g}\right)$ & $1.82 \pm 0.22$ & $2.25 \pm 0.14$ & $1.52 \pm 0.07^{\#}$ & $2.10 \pm 0.19$ \\
\hline
\end{tabular}

Values are presented as means \pm SEM. $n=6$ per group. EDL, extensor digitorum longus; Con, control; CSA, cross-sectional area; $D M$, diabetes; DM+HS, diabetes and heat stress; HS, heat stress. ${ }^{*} p<0.05, * * p<0.01$ and ${ }^{* * *} p<0.001$ vs. the Con group. ${ }^{\#} p<0.05$, ${ }^{\# \#} p<0.01$ and ${ }^{\# \# \#} p<0.001$ vs. the HS group. ${ }^{+} p<0.05$ vs. the DM group.

The fast-twitch muscle fiber CSA of the EDL was significantly decreased in the DM (47\% of Con, $p<0.001$ ) and DM+HS (58\% of Con, $p<0.001$ ) groups compared with that in the Con group. Compared with the Con group level, the fast-twitch muscle fiber CSA/body weight ratio was significantly decreased in the DM group (64\% of Con, $p<0.01$ ) but not in the DM+HS group $(p=0.55)$. Moreover, the fast muscle fiber CSA/body weight ratio was significantly lower in the DM group than in the DM+HS group ( $72 \%$ of DM+HS, $p=0.04$ ).

The slow-twitch muscle fiber CSA was significantly decreased in the DM group (62\% of Con, $p=0.016)$ but not in the DM+HS group $(p=0.17)$ compared with that in the Con group. This ratio was not decreased in the DM $(p=0.59)$ and DM+HS $(p=0.65)$ groups compared with the Con group ratio. These findings suggest that HS 
attenuates DM-induced skeletal muscle atrophy, especially that involving fast-twitch muscle fibers.

\section{Discussion}

The purpose of this study was to investigate the effect of HS on SDH activity in skeletal muscle of diabetic rats. The findings were as follows: 1) The decrease in SDH activity was significantly induced in the skeletal muscle by DM but attenuated by HS; 2) HSP60 level was significantly decreased in the skeletal muscle of diabetic rats, but it was maintained by HS; 3) SOD2 content was significantly decreased in the skeletal muscle of diabetic rats, but it was maintained by HS; and 4) skeletal muscle atrophy was induced by DM but attenuated by HS.

SDH activity in the EDL was decreased in diabetic rats compared with that in non-diabetic rats. Chen and Ianuzzo (1982) reported that the SDH activity decreased in the plantaris muscle of diabetic rats. Moreover, our data were consistent with their study. Interestingly, SDH activity in the EDL of heat-stressed diabetic rats was greater than that of the non-heat-stressed diabetic rats. HS has been reported to prevent mitochondrial dysfunction in denervated skeletal muscle in mice (Tamura et al. 2015), and our finding was similar. We hypothesized that HS would prevent reductions in SDH activity in the skeletal muscle of diabetic rats. Our finding supported our hypothesis, and this was the first study to suggest that HS can prevent the decrease in SDH activity in skeletal muscle induced by DM.

HSP60 appears to play a crucial role in the reduction of and resistance to oxidative stress. Silencing of HSP60 in several cell lines has been reported to increase ROS production (Kleinridders et al. 2013, Tang et al. 2016), decrease cell viability under oxidative stress (Tang et al. 2016) and induce mitochondrial dysfunction (Kleinridders et al. 2013). Moreover, HSP60-overexpressing rat small intestinal epithelial cells displayed increased cell viability under oxidative conditions (Takada et al. 2010). These findings suggest that HSP60 suppresses excessive ROS production and promotes resistance to oxidative stress and the maintenance of mitochondrial function. HSP60 content in the heart is decreased in diabetic rats (Shan et al. 2003, Chen et al. 2005), in line with our findings in skeletal muscle. Importantly, HSP60 content was significantly higher in the skeletal muscle of heat-stressed diabetic rats than in non-stressed diabetic rats, and its levels in the skeletal muscle of heat-stressed diabetic rats were similar to that in normal rat skeletal muscle. The decrease in HSP60 levels was expected to increase ROS production in the skeletal muscle of diabetic rats, whereas HS maintained HSP60 content, resulting in the prevention of excessive ROS production and resistance to oxidative stress in diabetic rat skeletal muscle.

SOD2 is expressed in mitochondria, and it reduces oxidative stress via the degradation of $\mathrm{O}_{2}$. Although SOD2 content was decreased in the skeletal muscle of diabetic rats, antioxidant administration prevented the decrease (Malardé et al. 2015, Guan et al. 2016). These findings suggest that oxidative stress decreases SOD2 content in skeletal muscle. In this study, DM was expected to increase oxidative stress in skeletal muscle because SOD2 content was also decreased in skeletal muscle. Importantly, HS prevented the decrease in SOD2 content in the skeletal muscle of diabetic rats, suggesting that HS decreased oxidative stress. Moreover, SOD2 overexpression has been reported to protect mitochondrial function in the hearts of diabetic mice (Shen et al. 2006), suggesting that SOD2 plays an important role in protecting mitochondrial function. Therefore, HS might prevent the decrease in SOD2 content by suppressing excessive oxidative stress, resulting in the prevention of the decrease in SDH activity in the skeletal muscle of diabetic rats.

DM-induced muscle atrophy has been suggested to be involved in oxidative stress (Nonaka et al. 2014). ROS production was increased in $\mathrm{C} 2 \mathrm{C} 12$ myotubes under high glucose conditions (Russell et al. 2009). Antioxidant administration decreased skeletal muscle atrophy in diabetic rats and mice (Mastrocola et al. 2008, Ono et al. 2015, Takada et al. 2015). These findings suggest that oxidative stress is involved in the mechanism of DM-induced skeletal muscle atrophy. We previously reported that HS attenuated DM-induced skeletal muscle atrophy (Nonaka et al. 2015). In this study, DM-induced skeletal muscle atrophy was also attenuated by HS. It is expected that the decrease in oxidative stress was one explanation for prevention of DM-induced skeletal muscle atrophy by HS.

The body weight was decreased in both the DM groups. STZ-induced DM rats have been reported to decrease body weight (Jacobs et al. 1998), and our data was corresponding with the previous study. HS exacerbated the loss of body weight in DM rats, suggesting that HS has a harmful effect on DM. HS has been reported to increase corticosterone and cortisol (Gong et al. 2015), which increase blood glucose level. 
In this study, blood glucose level induced by HS, corticosterone and cortisol were not measured. However, HS may increase blood glucose levels, resulting in inducing excessive loss of body weight in DM rats.

As described previously, the mechanism by which HS prevented the decrease in SDH activity in skeletal muscle was expected to involve a decrease in oxidative stress from the data, including changes in HSP60 and SOD2 content and improvement of skeletal muscle atrophy. However, we did not measure changes in antioxidant enzyme activities, including SOD2, glutathione peroxidase and catalase; oxidative stress markers and ROS production in the skeletal muscle. Future research is needed to determine whether HS alters these indicators of oxidative stress in the skeletal muscle mitochondria in diabetic rats. In addition, HS may have some harmful effects on DM. Therefore, the harmful effects induced by HS in DM, including changes in blood glucose level, and whether it can be suppressed in some manner should be clarified in detail.

In conclusion, the findings of this study suggest that HS can prevent the decrease in SDH activity in skeletal muscle associated with DM. However, HS also has a harmful effect, which enhances the body weight loss induced by DM.

\section{Conflict of Interest}

There is no conflict of interest.

\section{Acknowledgements}

This study was supported by JSPS KAKENHI Grant Number 26292073. The authors would like to thank Enago (www.enago.jp) for the English language review.

\section{References}

ARMSTRONG RB, GOLLNICK PD, IANUZZO CD: Histochemical properties of skeletal muscle fibers in streptozotocin-diabetic rats. Cell Tissue Res 162: 387-394, 1975.

CHEN HS, SHAN YX, YANG TL, LIN HD, CHEN JW, LIN SJ, WANG PH: Insulin deficiency downregulated heat shock protein 60 and IGF-1 receptor signaling in diabetic myocardium. Diabetes 54: 175-181, 2005.

CHEN V, IANUZZO CD: Metabolic alterations in skeletal muscle of chronically streptozotocin-diabetic rats. Arch Biochem Biophys 217: 131-138, 1982.

CHURCH TS, CHENG YJ, EARNEST CP, BARLOW CE, GIBBSONS LW, PRIEST EL, BLAIR SN: Exercise capacity and body composition as predictors of mortality among men with diabetes. Diabetes Care 27: 83-88, 2004.

GONG S, MIAO YL, JIAO GZ, SUN MJ, LI H, LIN J, LUO MJ, TAN JH: Dynamics and correlation of serum cortisol and corticosterone under different physiological or stressful conditions in mice. PLoS One 10: e0117503, 2015.

GUAN Y, CUI ZJ, SUN B, HAN LP, LI CJ, CHEN LM: Celastrol attenuates oxidative stress in the skeletal muscle of diabetic rats by regulating the AMPK-PGC1 $\alpha$-SIRT3 signaling pathway. Int J Mol Med 37: 1229-1238, 2016.

GÜRDAL A, KASIKCIOGLU E, YAKAL S, BUGRA Z: Impact of diabetes and diastolic dysfunction on exercise capacity in normotensive patients without coronary artery disease. Diab Vasc Dis Res 12: 181-188, 2015.

HE J, WATKINS S, KELLEY DE: Skeletal muscle lipid content and oxidative enzyme activity in relation to muscle fiber type in type 2 diabetes and obesity. Diabetes 50: 817-823, 2001.

ISHIHARA A, NAGATOMO F, TERADA M, FUJINO H, KONDO H, ISHIOKA N: Effects of microgravity on the mouse triceps brachii muscle. Muscle Nerve 52: 63-68, 2015.

JACOBS RL, HOUSE JD, BROSNAN ME, BROSNAN JT: Effects of streptozotocin-induced diabetes and of insulin treatment on homocysteine metabolism in the rat. Diabetes 47: 1967-1970, 1998.

KLEINRIDDERS A, LAURITZEN HP, USSAR S, CHRISTENSEN JH, MORI MA, BROSS P, KAHN CR: Leptin regulation of Hsp60 impacts hypothalamic insulin signaling. J Clin Invest 123: 4667-4680, 2013.

LITTLE JP, GILLEN JB, PERCIVAL ME, SAFDAR A, TARNOPOLSKY MA, PUNTHAKEE Z, JUNG ME, GIBALA MJ: Low-volume high-intensity interval training reduces hyperglycemia and increases muscle mitochondrial capacity in patients with type 2 diabetes. $J$ Appl Physiol (1985) 111: 1554-1560, 2011.

LUSTGARTEN MS, JANG YC, LIU Y, QI W, QIN Y, DAHIA PL, SHI Y, BHATTACHARYA A, MULLER FL, SHIMIZU T, SHIRASAWA T, RICHARDSON A, VAN REMMEN H: MnSOD deficiency results in elevated oxidative stress and decreased mitochondrial function but does not lead to muscle atrophy during aging. Aging Cell 10: 493-505, 2011. 
MALARDÉ L, GROUSSARD C, LEFEUVRE-ORFILA L, VINCENT S, EFSTATHIOU T, GRATASDELAMARCHE A: Fermented soy permeate reduces cytokine level and oxidative stress in streptozotocininduced diabetic rats. $J$ Med Food 18: 67-75, 2015.

MASTROCOLA R, REFFO P, PENNA F, TOMASINELLI CE, BOCCUZZI G, BACCINO FM, ARAGNO M, COSTELLI P: Muscle wasting in diabetic and in tumor-bearing rats: Role of oxidative stress. Free Radic Biol Med 44: 584-593, 2008.

NONAKA K, UNE S, AKIYAMA J: Heat stress attenuates skeletal muscle atrophy of extensor digitorum longus in streptozotocin-induced diabetic rats. Acta Physiol Hung 102: 293-300, 2015.

NONAKA K, UNE S, TATSUTA N, ITO K, AKIYAMA J: Changes in antioxidant enzymes and lipid peroxidation in extensor digitorum longus muscles of streptozotocin-diabetic rats may contribute to muscle atrophy. Acta Physiol Hung 101: 421-428, 2014.

OISHI Y, TANIGUCHI K, MATSUMOTO H, ISHIHARA A, OHIRA Y, ROY RR: Muscle type-specific response of HSP60, HSP72, and HSC73 during recovery after elevation of muscle temperature. J Appl Physiol (1985) 92: 1097-1103, 2002.

ONO T, TAKADA S, KINUGAWA S, TSUTSUI H: Curcumin ameliorates skeletal muscle atrophy in type 1 diabetic mice by inhibiting protein ubiquitination. Exp Physiol 100: 1052-1063, 2015.

OKITA K, YONEZAWA K, NISHIJIMA H, HANADA A, OHTSUBO M, KOHYA T, MURAKAMI T, KITABATAKE A: Skeletal muscle metabolism limits exercise capacity in patients with chronic heart failure. Circulation 98: 1886-1891, 1998.

PERRY RA JR, BROWN LA, LEE DE, BROWN JL, BAUM JI, GREENE NP, WASHINGTON TA: The Akt/mTOR pathway: Data comparing young and aged mice with leucine supplementation at the onset of skeletal muscle regeneration. Data Brief 8: 1426-1432, 2016.

POWERS SK, WIGGS MP, DUARTE JA, ZERGEROGLU AM, DEMIREL HA: Mitochondrial signaling contributes to disuse muscle atrophy. Am J Physiol Endocrinol Metab 303: E31-E39, 2012.

RUSSELL ST, RAJANI S, DHADDA RS, TISDALE MJ: Mechanism of induction of muscle protein loss by hyperglycaemia. Exp Cell Res 315: 16-25, 2009.

SHAN YX, YANG TL, MESTRIL R, WANG PH: Hsp10 and Hsp60 suppress ubiquitination of insulin-like growth factor-1 receptor and augment insulin-like growth factor-1 receptor signaling in cardiac muscle: Implications on decreased myocardial protection in diabetic cardiomyopathy. J Biol Chem 278: 45492-45498, 2003.

SHEN X, ZHENG S, METREVELI NS, EPSTEIN PN: Protection of cardiac mitochondria by overexpression of MnSOD reduces diabetic cardiomyopathy. Diabetes 55: 798-805, 2006.

SOUKUP T, ZACHAROVÁ G, SMERDU V: Fibre type composition of soleus and extensor digitorum longus muscles in normal female inbred Lewis rats. Acta Histochem 104: 399-405, 2002.

TAKADA M, OTAKA M, TAKAHASHI T, IZUMI Y, TAMAKI K, SHIBUYA T, SAKAMOTO N, OSADA T, YAMAMOTO S, ISHIDA R, ODASHIMA M, ITOH H, WATANABE S: Overexpression of a 60-kDa heat shock protein enhances cytoprotective function of small intestinal epithelial cells. Life Sci 86: 499-504, 2010.

TAKADA S, KINUGAWA S, MATSUSHIMA S, TAKEMOTO D, FURIHATA T, MIZUSHIMA W, FUKUSHIMA A, YOKOTA T, ONO Y, SHIBATA H, OKITA K, TSUTSUI H: Sesamin prevents decline in exercise capacity and impairment of skeletal muscle mitochondrial function in mice with high-fat diet-induced diabetes. Exp Physiol 100: 1319-1330, 2015.

TAMURA Y, KITAOKA Y, MATSUNAGA Y, HOSHINO D, HATTA H: Daily heat stress treatment rescues denervation-activated mitochondrial clearance and atrophy in skeletal muscle. J Physiol 593: 2707-2720, 2015.

TANG H, CHEN Y, LIU X, WANG S, LV Y, WU D, WANG Q, LUO M, DENG H: Downregulation of HSP60 disrupts mitochondrial proteostasis to promote tumorigenesis and progression in clear cell renal cell carcinoma. Oncotarget 5: 38822-38834, 2016.

TRYON LD, VAINSHTEIN A, MEMME JM, CRILLY MJ, HOOD DA: Recent advances in mitochondrial turnover during chronic muscle disuse. Integr Med Res 3: 161-171, 2014. 
YOKOTA T, KINUGAWA S, OKITA K, HIRABAYASHI K, SUGA T, HATTORI M, NAKAGAWA Y, OYAMA-MANABE N, SHIRATO H, TSUTSUI H: Lower aerobic capacity was associated with abnormal intramuscular energetics in patients with metabolic syndrome. Hypertens Res 34: 1029-1034, 2011. 